\title{
The level of superoxide dismutase and catalase in acyanotic congenital heart disease children with heart failure
}

Henry Wicaksono *, Mahrus A Rahman, Roedi Irawan, I Ketut Alit Utamayasa, Teddy Ontoseno and Taufiq Hidayat

Department of Child Health, Faculty of Medicine, Universitas Airlangga / Dr. Soetomo General Hospital, Surabaya, Indonesia.

GSC Biological and Pharmaceutical Sciences, 2021, 16(01), 150-156

Publication history: Received on 18 June 2021; revised on 22 July 2021; accepted on 24 July 2021

Article DOI: https://doi.org/10.30574/gscbps.2021.16.1.0206

\begin{abstract}
Background: The most common complication in acyanotic congenital heart disease (CHD) is heart failure which definitive diagnosis and therapy remain unsatisfactory. Heart failure's progression is often associated with oxidative stress process. Superoxide dismutase (SOD) is the first line antioxidant of defense against superoxide anion. While Catalase (CAT) breaks down hydrogen peroxide into water and oxygen molecules which complements previous detoxification carried out by SOD.
\end{abstract}

Objective: This study aimed to compare the differences of SOD and CAT levels in acyanotic CHD patients between those with and without heart failure.

Methods: A case-control study was conducted on three to ten years old children with a left-to-right shunt acyanotic CHD with and without heart failure in the Pediatric Cardiology outpatient clinic, ward, and emergency room of Dr. Soetomo Hospital Surabaya from April-July 2020. Echocardiography was used to establish the diagnosis of CHD, while Pediatric Heart Failure Score (PHFS) criteria was used to indicate heart failure. T-test was undertaken for analysing the difference between both groups.

Results: The total samples were 41 children, consisted of 29 subjects in the case group (CHD with heart failure) and 12 subjects in the control group (without heart failure). The level of SOD in CHD with heart failure was lower $(74.670 \pm 15.705)$ than those without it $(109.163 \pm 3.111)(p<0.05)$. In contrast, level of CAT in CHD with heart failure was higher (25.895) than those without it $(13.976)(p<0.05)$.

Conclusion: There was a significant difference of SOD and CAT levels in acyanotic CHD between those with and without heart failure.

Keywords: SOD; CAT; Acyanotic CHD; Heart failure; Oxidative stress; Children

\section{Introduction}

One of the most common complications in CHD is heart failure that closely relates to ventricular dysfunction and overload of volume and pressure [1]. The incidence of heart failure in CHD is about $0.8 \%$ of live births [2,3]. There are about 12,000-35,000 cases of heart failure that causes about 11,000-14,000 cases of hospitalization for children aged

\footnotetext{
${ }^{*}$ Corresponding author: Henry Wicaksono

Department of Child Health, Faculty of Medicine, Universitas Airlangga / Dr. Soetomo General Hospital, Surabaya, Indonesia. 
less than 19 years in the United States each year [4]. Less than 50\% of children with symptomatic heart failure have a 5 -year survival without heart transplant that is influenced by the timing of initial diagnosis and treatment $[5,6]$.

Heart failure is defined as a clinical syndrome caused by either inability to pump enough blood throughout the body as needed or unable to return pulmonary or systemic blood flow or the combination of both [7]. According to Ross, it is a result of disorders of the structure and function of the heart that affect the ability of the ventricles to fill and/or pump blood [8]. According to Nandi and Rossano, heart failure, regardless of the etiology, is generally defined as a syndrome of clinical and pathophysiological symptoms caused by impaired filling or blood pump in the ventricles that causes inadequate organ perfusion. Heart failure in children is a complex disease process that can occur secondary to various underlying etiologies, such as congenital heart disease, cardiomyopathy, and/or other acute conditions [4].

Until now, the definitive diagnosis and therapy of heart failure in children with CHD remain unsatisfactory. Recently, the progression of heart failure in CHD has been associated with oxidative stress process approach. Moreover, increasing production of reactive oxygen species (ROS) is associated with the emergence of both pulmonary and systemic vascular disorders resulting in heart failure [9].

The production of free radical molecules, such as $\mathrm{O}_{2}-$ and $\mathrm{H}_{2} \mathrm{O}_{2}$ can cause several mechanisms. First, activation of redox sensitive kinase which will cause inflammation and angiogenesis. Second, inactivation of protein phosphatase to modulate redox-sensitive signaling will lead to hypertrophy, proliferation, and migration of endothelial cells. Third, activation of redox-sensitive transcription factors will cause proinflammatory gene expression (VCAM1 Vascular Cell Adhesion Molecule 1, MCP1 Monocyte chemoattractant protein 1). Fourth, modulate calcium ion channels (Ca ${ }^{2+}$ ) have an effect on vascular remodeling, inflammation, increasing vascular permeability, and angiogenesis. Fifth, activation of matrix metalloproteinases (MMPs) will cause extracellular matrix remodeling [10].

Apart from $\mathrm{O}_{2}-$ and $\mathrm{H}_{2} \mathrm{O}_{2}$, nitric oxide synthase (NOS) has a role in the pathophysiological mechanisms of heart disease. The formation of NOS begins with the nitric oxide (NO) molecule that quickly reacts with $\mathrm{O}_{2}-$ with the help of the enzymes NADPH oxidase, xanthine oxidase, and mitochondria to form peroxynitrite anion (ONO0- Peroksinitrit), of which will oxidize several molecules, including heme from soluble guanylate cyclase (sGC), lipids, and endothelial nitric oxide synthase (eNOS). These reactions will result in vascular inflammation, changes in vascular tone, increasing vascular permeability, and increasing platelet aggregation [10].

Vascular superoxide $\left(\mathrm{O}_{2}^{-}-{ }^{-}\right.$is normally produced by the product of cellular metabolism and will differ it to form other types of ROS, such as hydrogen peroxide $\left(\mathrm{H}_{2} \mathrm{O}_{2}\right)$ dan Peroxynitrite (ONOO-). In blood vessels, changes in superoxide levels will affect vascular tone modulation, gene expression, inflammation, cell growth, signaling, and apoptosis. Superoxide dismutase as the first line against ROS in living cells will catalyze the reduction and oxidation reactions from superoxide to hydrogen peroxide and oxygen molecules. If there are reduced transition metals $\left(\mathrm{Fe}^{2+}, \mathrm{Cu}^{2+}\right), \mathrm{H}_{2} \mathrm{O}_{2} \mathrm{can}$ undergo a spontaneous conversion to hydroxyl radicals $(\mathrm{OH} \cdot)$ or other types of ROS that are highly reactive. Nitric oxide (anti-inflammatory, anticoagulant, and vasodilator) can also be inactivated very quickly when it reacts with $\mathrm{O}_{2}-$ and causes the production of the strong oxidant peroxynitrite (ONOO-) and endothelial and mitochondrial dysfunction. Therefore, SOD is the first line of defense against the toxicity of anionic superoxide radicals. SOD activity requires metal catalysis. Meanwhile, Catalase (CAT) has a function to break down hydrogen peroxide molecules into water and oxygen molecules that complement the previous detoxification process carried out by SOD [10].

Superoxide Dismutase (SOD) and Catalase (CAT) are type of antioxidant produced in the mitochondria of heart muscle that work against excessive ROS production. Basic knowledge about the levels of SOD and CAT in children with CHD acyanotic left to right shunts to determine the oxidative stress process that occurs in heart failure as a basis for innovative diagnosis and therapy of heart failure in CHD patients in the future is necessary [10].

\section{Material and methods}

This study was an analytical observational research with a case-control design to compare SOD and CAT levels in leftto-right shunt acyanotic CHD between those with heart failure and without heart failure. The samples were all children aged 3-10 years old with a left-to-right shunt acyanotic CHD with heart failure and those without heart failure who came through Emergency room (ER) and the Pediatric Cardiology Outpatient Clinic (OPC) in the period after obtaining permission and approval from the Health Research Ethics Committee at Dr. Soetomo Hospital Surabaya. Informed consent was obtained from all individual participants included in the study. They were chosen using consecutive nonrandom sampling. The definitive diagnosis of acyanotic CHD was determined based on echocardiographic examination by pediatric cardiology consultant. Types of heart defects categorized as CHD acyanotic left-to-right shunt here were 
ventricular septal defect (VSD), atrial septal defect (ASD), and patent ductus arteriosus (PDA). While the diagnosis of heart failure was established by Pediatric Heart Failure Score (PHFS) criteria.

Inclusion criteria were children with left-to-right shunt acyanotic CHD aged 3-10 years old who meet the clinical criteria for heart failure according to PHFS with a score more than 2 for the case group. While the exclusion criteria were children with left-to-right shunt acyanotic CHD who had cardiac surgery plan within the next one month, impaired kidney function with decreased Glomerular Filtration Rate (GFR) according to age or with renal replacement therapy, malignancy, hyperkalemia with potassium levels more than $5.5 \mathrm{mEq} / \mathrm{L}$ or hypokalemia with potassium levels less than $3 \mathrm{mEq} / \mathrm{L}$, history of metabolic and hormonal diseases (e.g. diabetes mellitus, hypothyroidism, hyperthyroidism, and congenital adrenal hyperplasia), girls who had menstruated, diseases affecting the vascular (e.g vasculitis, hypertension, and cerebrovascular disease), and critically-ill (getting intravenous inotropes, pneumonia on a ventilator, terminal conditions, and sepsis).

Tool to check the level of SOD from blood was the Superoxide Dismutase Typed Activity Kit (Elabscience paint \# EBCK022-S). Sampling was $1 \mathrm{ml}$ venous blood plus citrate/EDTA, then centrifuged for 10 minutes at $40^{\circ} \mathrm{C}$. Plasma layer was transferred to a new tube and stored at $80^{\circ} \mathrm{C}$ then analyzed with a $450 \mathrm{~nm}$ ELISA kit microplate reader. The level of SOD was expressed in units of U/ml. The HUMAN Catalase assay Kit (Cayman Chem. Paint \# 707002) was used to check CAT level. Sampling was $0.2 \mathrm{ml}$ erythrocytes in $0.2 \mathrm{ml}$ cold assay buffer then centrifuged for 15 minutes at $4^{\circ} \mathrm{C}$. The supernatant was taken and kept in ice, measured by the calorimetric method at $570 \mathrm{~nm}$. Level of CAT was expressed in $\mu \mathrm{M}$ units.

All data were analyzed using SPSS version 20.0. The basic characteristics of research subjects (e.g. age, gender, nutritional status, and type of CHD) were presented descriptively in tabular form. To find out whether there were differences in SOD and CAT levels between the case and control groups, the data normality test was firstly conducted with Kolomogorov-Smirnov test. If the data were normally distributed ( $p>0.05)$, the data were tested with an independent sample T-test, but if not $(\mathrm{p}<0.05)$, the data were tested using a Mann-Whitney test.

The ethical clearance was approved by the Ethical Committee of Dr. Soetomo General Hospital Surabaya (No.1198/KEPK/V/2019).

\section{Results}

Initial sampling was conducted from April 2019 to March 2020. During the study periods, there were six subjects resigned because they refused to sign an informed consent.

Table 1 Characteristic of samples

\begin{tabular}{|c|c|c|c|}
\hline \multirow[b]{2}{*}{ Variables } & \multicolumn{2}{|c|}{ Percentage (\%) } & \multirow[b]{2}{*}{$p$} \\
\hline & $\begin{array}{l}\text { CHD with Heart } \\
\text { Failure }(\mathrm{N}=29)\end{array}$ & $\begin{array}{l}\text { CHD without Heart } \\
\text { Failure }(\mathrm{N}=12)\end{array}$ & \\
\hline \multicolumn{4}{|l|}{ Sex } \\
\hline Male & $13(45 \%)$ & $6(50 \%)$ & 0.763 \\
\hline Female & $16(55 \%)$ & $6(50 \%)$ & \\
\hline Median age (years) & $9(2-14)$ & $8.5(2-14)$ & 0.806 \\
\hline \multicolumn{4}{|l|}{ Nutritional Status } \\
\hline $\begin{array}{l}\text { Normal } \\
\text { Moderate }\end{array}$ & $\begin{array}{c}24(83 \%) \\
5(17 \%)\end{array}$ & $\begin{array}{c}12(100 \%) \\
0(0 \%)\end{array}$ & 0.125 \\
\hline \multicolumn{4}{|l|}{ Type of CHD } \\
\hline VSD & 17 (59\%) & $6(50 \%)$ & 0.113 \\
\hline PDA & $12(41 \%)$ & $3(25 \%)$ & \\
\hline ASD & 0 & 3 & \\
\hline
\end{tabular}


Therefore, at the end of the study, the total sample that met the inclusion criteria was 41 subjects, consisted of 29 subjects in the case group (CHD with heart failure) and 12 subjects in the control group (CHD without heart failure). Descriptive analysis was carried out to see the frequency and distribution of variables. Table 1 shows the characteristics of children.

There was no statistically significant difference in the proportion of sex between the two groups although the number of girl in case group was slightly higher than those in control group. In the case and control groups, the median age values were 9 and 8.5 years, respectively. There was no statistically significant difference in the age variable between the two groups. Most of the subjects in both groups were still in normal nutritional status. Ventricular Septal Defect (VSD) was still the most common type of CHD in both groups.

Table 2 The level of SOD dan CAT between CHD accompanied by heart failure and without heart failure

\begin{tabular}{|l|l|l|l|}
\hline \multirow{2}{*}{ Variables } & \multicolumn{2}{|c|}{ Acyanotic CHD } & \multirow{2}{*}{ p } \\
\cline { 2 - 3 } & $\begin{array}{c}\text { with heart failure } \\
(\mathbf{N}=\mathbf{2 9})\end{array}$ & $\begin{array}{c}\text { without heart failure } \\
(\mathbf{N}=\mathbf{1 2})\end{array}$ & \\
\hline Mean SOD $(\mathrm{U} / \mathrm{mL})$ & $74.670( \pm 15.705)$ & $109.163( \pm 3.111)$ & $0.001^{*}$ \\
\hline Median CAT $(\mu \mathrm{M})$ & $25.895(6.070-70.230)$ & $13.976(10.430-16.419)$ & $0.002^{* *}$ \\
\hline
\end{tabular}

In the SOD variable, after the normality test was carried out with Kolomogorov-Smirnov test, it was found that the data were normally distributed in both groups ( $\mathrm{p}>0.05)$, therefore the numerical data were presented in the form of a mean accompanied by a standard deviation value. The mean SOD levels in CHD patients with heart failure were lower (74,670 $\pm 15,705)$ than those without heart failure $(109.163 \pm 3.111)$. The data were then analyzed by parametric test using independent sample T-test. As a result, there was a significant difference in SOD levels in the two groups $(p<0.05)$.

On the contrary, the CAT variable data were not normally distributed $(p<0.05)$, therefore the numerical data were presented in the form of the median accompanied by the maximum and minimum values. The median CAT results in patients with CHD with heart failure were higher $(25,895)$ than those without heart failure (13.976). The data were then analyzed by non-parametric test using the Mann-Whitney. As a result, there was also a significant difference in CAT levels in the two groups $(p<0.05)$.

\section{Discussion}

In this study, SOD level in children with acyanotic left-to-right shunts with heart failure was lower than those without heart failure. Superoxide dismutase is basically the first line of antioxidant defense against the process of oxidative stress formation [10]. This enzyme has an important role in superoxide metabolism because of its closest location to the main source of producing ROS, mitochondria [11]. If there is a decrease in SOD levels, this oxidative stress activity will cause a series of processes that lead to endothelial damage and remodeling of the heart muscle then ultimately will lead to heart failure. Moreover, recent evidence suggests that at each subcellular location, SODs catalyze the conversion of $\mathrm{O}_{2}-$ and $\mathrm{H}_{2} \mathrm{O}_{2}$ that have a role in cell communication. It also plays an important role in inhibiting the oxidative inactivation of NO thus preventing peroxynitrit formation and also endothelial and mitochondrial dysfunction. In normal cellular metabolic processes, oxygen undergoes a series of univalent reductions that cause the formation of $\mathrm{O}_{2}$, hydrogen peroxide $\left(\mathrm{H}_{2} \mathrm{O}_{2}\right)$, and $\mathrm{H}_{2} \mathrm{O}$. Potential sources of ROS-forming enzymes include components of the electron transport chain, xanthine oxidase, cytochrome p450 monooxygenase, lipoxygenase, NOS, and NADPH oxidase. Anion superoxide is dismutated by SOD into $\mathrm{H}_{2} \mathrm{O}_{2}$ and then catalyzed into $\mathrm{H}_{2} \mathrm{O}$ by the enzyme catalase, peroxiredoxins (Prxs), or glutathione peroxidase (GPx) [10].

The result of this study is consistent with a case control study conducted by Gao-Zhong et al in 2010 for children aged 1 month to 3 years with left-to-right shunt acyanotic CHD. The level of SOD with heart failure was found to be lower than the control group ( $\mathrm{p}<0.01)$. Gao-Zhong concluded that the process of oxidative stress was the cause of incidence of left-to-right shunt CHD accompanied by heart failure [12].

On the contrary, CAT levels in children with left-to-right acyanotic CHD who experienced heart failure in the present study were found to be higher than those without heart failure. Unlike SOD, Catalase enzymes are not the first line of antioxidant enzymes to fight ROS. This enzyme uses iron or manganese as a cofactor and serves to catalyze the 
degradation or reduction of hydrogen peroxide $\left(\mathrm{H}_{2} \mathrm{O}_{2}\right)$ into water $\left(\mathrm{H}_{2} \mathrm{O}\right)$ and oxygen $\left(\mathrm{O}_{2}\right)$ molecules that will complement the previous detoxification process carried out by SOD. This enzyme works effectively by breaking down millions of hydrogen peroxide molecules in seconds [13]. In carrying out its function, CAT is assisted by the enzyme Glutathione Peroxidase (GPx) and Peroxiredoxin. Based on its chemical characteristic, GPx has a much higher affinity ability to bind hydrogen peroxide compared to CAT [14]. This may be the reason why CAT levels in patients with leftto-right shunt acyanotic CHD who experienced heart failure in this study remained higher than those without heart failure. However, in this study, the levels of GPx and Peroxiredoxin were not measured.

Furthermore, excessive ROS caused proliferation of myocardial fibroblasts by remodeling the heart muscle. This result leads to the hypothesis that oxidative stress may be the next target of therapy in heart failure patients. Clinical trials have begun to study the effects of antioxidant therapy in humans with heart failure. Oxidative stress treatment itself can have a different approach, such as inhibition of oxidative stress production, increasing endogenous antioxidant capacity by administering exogenous antioxidants, covering vitamin C, vitamin A, vitamin E, and folic acid [15].

\section{Limitations}

Case-control study is observational and does not provide the same level of evidence as randomized controlled trials and meta-analyzes. The effect of external variables is technically difficult to control and measure due to the large number of confounding factors, for example hormonal factors and endogenous antioxidant content (e.g., vitamins A, C, and E) or nonenzymatic components including glutathione, metallothionein, a-tocopherol, and ascorbate. Heart failure is a complex disorder. There are many compensatory mechanisms for overcoming inadequate perfusion of vital organs. Impaired local circulation of important organs along with endothelial and neurohormonal dysfunction are associated with oxidant-antioxidant imbalance and subcellular disorders.

\section{Research Strength}

The present study is one of the few studies revealing the differences of SOD and CAT levels in pediatric patients with CHD who experienced heart failure. Previous studies had been more frequently conducted and focused on experimental animals and adult.

Table 3 Pediatric heart failure score

\begin{tabular}{|l|l|l|l|}
\hline & \multicolumn{1}{|c|}{$\mathbf{0}$} & \multicolumn{1}{|c|}{$\mathbf{1}$} & \multicolumn{1}{|c|}{$\mathbf{1}$} \\
\hline Diaphoresis & Head only & Head and body during exercise & Head and body at rest \\
\hline Tachypnea & Rare & Several times & Frequent \\
\hline Breathing & Normal & Retractions & Dyspnea \\
\hline Respiratory rate & & & $>60$ \\
\hline$* 0-1$ year & $<50$ & $50-60$ & $>45$ \\
\hline$* 1-6$ years & $<35$ & $35-45$ & $>35$ \\
\hline$* 7-10$ years & $<25$ & $25-35$ & $>28$ \\
$* 11-14$ years & $<18$ & $18-28$ & $>170$ \\
\hline $\begin{array}{l}\text { Heart rate } \\
\text { x/mnt) }\end{array}$ & & & $>115$ \\
\hline$* 0-1$ year & $<105$ & $160-170$ & $>100$ \\
\hline$* 1-6$ years & $<90$ & $90-115$ & $>90$ \\
\hline$* 7-10$ years & $<80$ & $80-90$ & $>3$ \\
\hline$* 11-14$ years & $<2$ & $2-3$ & \\
\hline $\begin{array}{l}\text { Liver edge from } \\
\text { costal margin } \\
\text { (cm) }\end{array}$ & & & \\
\hline
\end{tabular}




\section{Conclusion}

There is a significant difference of SOD and CAT levels in a left to right shunt acyanotic CHD between those with heart failure and without it. The level of SOD in children with heart failure is lower than those without heart failure. While CAT level in children with heart failure is higher than those without heart failure. The process of oxidative stress is the ultimate cause of incidence of heart failure in children with left-to-right shunt CHD.

\section{Compliance with ethical standards}

\section{Acknowledgments}

The authors thank the Director of Dr. Soetomo General Hospital, Surabaya Indonesia for supporting this research.

\section{Disclosure of conflict of interest}

"The Authors declare that there is no conflict of interest."

\section{Statement of ethical approval}

The ethical clearance was approved by the Ethical Committee of Dr. Soetomo General Hospital Surabaya (No.1198/KEPK/V/2019). Informed consent was obtained from all individual participants included in the study.

\section{Statement of informed consent}

Informed consent was obtained from all individual participants included in the study.

\section{References}

[1] Hinton RB, Ware SM. Heart failure in pediatric patients with congenital heart disease. Circ Res. 2017; 15: 978994.

[2] Lipshultz SE, Sleeper LA, Towbin JA, Lowe AM, Orav EJ, Cox GF. The incidence of pediatric cardiomyopathy in two regions of the United States. N Engl J Med. 2003; 348: 1647-1655.

[3] Rossano JW, Kim JJ, Decker JA, Price JF, Zafar F, Graves DE. Prevalence, morbidity, and mortality of heart failurerelated hospitalizations in children in the United States: a population-based study. J Card Fail. 2012; 18: 459470 .

[4] Nandi D, Rossano JW. Epidemiology and cost of heart failure in children. Cardiol Young. 2015; 25(8): 1460-1468.

[5] Kantor PF, Lougheed J, Dancea A, McGillion N, Barbosa N, Chan C, et al. Presentation, diagnosis, and medical management of heart failure in children: Canadian cardiovascular society guidelines. Can J Cardiol. 2013; 29(12): 1535-1552.

[6] Towbin JA, Lowe AM, Colan SD, Sleeper LA, Orav EJ, Clunie S. Incidence, causes, and outcomes of dilated cardiomyopathy in children. JAMA. 2006; 296(15): 1867-1876.

[7] Park MK. Park's pediatric cardiology for practitioners. 6th ed. Elsivier, Texas. 2021.

[8] Ross RD. The Ross classification for heart failure in children after 25 years: a review and an age-stratified revision. Pediatr Cardiol. 2012; 33(8): 1295-1300.

[9] Sharma S, Grobe AC, Wiseman DA, Kumar S, Englaish M, Najwer I, et al. Lung antioxidant enzymes are regulated by development and increased pulmonary blood flow. Lung Cell Mol Physiol. 2007; 293: 960-971.

[10] Fukai T, Ushio M. Superoxide dismutases: role in redox signaling, vascular function, and diseases. Antioxidants \& Redoks Signaling. 2011; 15(6): 1-24.

[11] Mendez JI, Nicholson WJ, Taylor WR. SOD isoforms and signalling in blood vessels: evidence for the importance of ROS compartmentalization. Arterioscler Thromb Vasc Biol. 2005; 25: 887-888.

[12] Gao-Zhong L, Dong XY, Yang S, Chen YQ, Lu JP. Erythrocyte oxidative stress in children with left to right shunt congenital heart disease. Int J Contemp Pediatr. 2010; 12: 440-443. 
GSC Biological and Pharmaceutical Sciences, 2021, 16(01), 150-156

[13] Ighodaro OM and Akinloye OA. First line defence antioxidants-superoxide dismutase (SOD), catalase (CAT) and glutathione peroxidase (GPX): their fundamental role in the entire antioxidant defence Grid. Alex J Med. 2018; 54(4): 287-293.

[14] Tsutsui H, Kinugawa S, and Matsushima S. Oxidative stress and heart Failure. Am J Physiol Heart Circ Physiol. 2011; 301: 2181-2190.

[15] Diaconu C. Oxidative stress and heart failure. Arch Balk Med Union. 2019; 54(2): 219-221. 